\title{
Construcción social del riesgo de desastre, colonia Puerto Príncipe, Nueva Guinea, RACCS, 2013
}

\author{
Social construction of disaster risk, Puerto Príncipe Community, Nueva Guinea, RACCS, 2013
}

\author{
Bismarck Abraham Castañeda Flores' \\ Mibsam Aragón Gutiérrez²
}

\section{Resumen}

Zn esta investigación se analizó el proceso de construcción social del riesgo de desastre ante inundaciones provocadas por lluvias intensas en la colonia Puerto Príncipe. Se indagó acerca del conocimiento que posee la población sobre el tema de desastre por inundaciones y las formas en las cuales la comunidad percibe las amenazas socio-naturales. Se revisaron las acciones individuales y colectivas que contribuyen al desarrollo de estrategias de adaptación social al riesgo, las formas de respuesta al riesgo en la práctica o en su imaginación y el rol de las organizaciones sociales en la definición e implementación de mecanismos comunitarios de adaptación a las inundaciones. Los resultados del estudio evidenciaron la existencia de diferentes contextos de vulnerabilidad que incrementan el riesgo de desastre en la colonia Puerto Príncipe.

Palabras clave: riesgo; vulnerabilidad; construcción social del riesgo; lluvias intensas; desastre; Puerto Príncipe.

\section{Summary}

In this investigation the process of social construction of the disaster risk in floods situations caused by intense rains in Puerto Príncipe Community was analyzed. It inquired about the knowledge that the population has about the subject of flood disasters and the ways in which the community perceives the socio-natural threats. It was reviewed the individual and collective actions that contribute to the development of social adaptation strategies to risk, the forms of response to risk in practice or in their imagination and the role of social organizations in the definition and implementation of community mechanisms of adaptation to floods. The results of the study showed the existence of different contexts of vulnerability that increase the risk of disaster in Puerto Príncipe Community.

Key Words: risk; vulnerability; Socila construction of risk; intense rains; disaster; Puerto Príncipe.

\section{Introducción}

Esta investigación analiza el proceso de construcción social del riesgo de desastre en la colonia Puerto Príncipe, ante las amenazas por inundaciones provocadas por lluvias intensas, en el 2013. El propósito del estudio fue determinar la construcción social del riesgo de desastre a partir de que el riesgo y el desastre son fenómenos socioculturales derivados de la relación naturaleza, cultura y desarrollo. Se procuró construir, deconstruir y reconstruir los principales valores, normas, mitos y creencias relacionados al riesgo de desastre en la comunidad. Todos los aspectos abordados en el estudio guardan una intrínseca relación

\footnotetext{
1 Licenciado en Sociología con Mención en Autonomía por la Universidad de las Regiones Autónomas de la Costa Caribe Nicaragüense-Recinto Universitario Nueva Guinea. Correo: biscasta@yahoo.com

2 Doctora en Educación con Especialidad en Mediación Pedagógica. Profesora de la Universidad de las Regiones Autónomas de la Costa Caribe Nicaragüense-Recinto Universitario Nueva Guinea: Correo: mibsamaragon@yahoo.com ORCID: https://orcid.org/0000-0001-6864-410X
} 
con los objetivos planteados en la investigación. Esta investigación permitirá la comprensión holística del riesgo como fenómeno sociocultural, y el impacto de las interrelaciones entre la comunidad y el medio ambiente en el desarrollo local, de igual manera permitirá a las autoridades y líderes comunitarios desarrollar estrategias efectivas de intervención institucional y de respuesta comunitaria al riesgo de desastre, de incrementar la capacidad de la comunidad para recuperarse autónomamente de los impactos provocados por las amenazas socionaturales.

Las recomendaciones señaladas en este estudio se ajustan a los hallazgos encontrados en la comunidad y están dirigidas, tanto a la comunidad en general como a los líderes comunitarios. Cada una de esas recomendaciones está orientada a tratar de subsanar las formas de percepción del riesgo de desastre y los diferentes contextos de vulnerabilidad construidos socialmente en la colonia.

\section{Revisión de literatura}

\section{Construcción social del riesgo de desastre}

Desde la perspectiva sociológica el desastre es considerado como tal cuando afecta sociedades vulnerables que no cuentan con la capacidad para enfrentarlo adecuadamente o recuperarse autónomamente de sus efectos. Fritz, Quarantelli, \& Kreps (citados por Fernández, 1996) definen el desastre desde el punto de vista sociológico, como:

... una ocasión de crisis o estrés social, observable en el tiempo y el espacio, en que sociedades o sus componentes (comunidades, regiones, etc.) sufren daños o pérdidas físicas y alteraciones en su funcionamiento rutinario, a tal grado que exceden su propia capacidad de autorecuperación, requiriendo la intervención o cooperación externa. Tanto las causas como las consecuencias de los desastres son producto de los procesos sociales que operan al interior de la sociedad afectada (pp. 19, 20).

Blaikie, Wilches Chaux, \& Maskrey (citados por Llavel, 2000) exponen el componente social del desastre materializado en sociedades o grupos sociales sometidos a procesos desiguales de desarrollo.
El desastre se convierte en un producto social derivado del desajuste de la relación entre la sociedad y la naturaleza (Llavel, 2000), señala “... los desastres son el producto de procesos de transformación y crecimiento de la sociedad, que no garantizan una adecuada relación con el ambiente natural y construido que le da sustento" (p.6).

Se deduce entonces, que la construcción social del riesgo de desastre se refiere a dos condiciones básicas, en primer lugar, a:

La construcción de contextos de vulnerabilidad, los cuales significan un desajuste o desadaptación al medio físico a tal grado que éste se convierte en una amenaza y, en consecuencia, en un factor de generación de riesgo que excede el riesgo aceptable (Rodríguez, 2007, pp. 91-92).

En segundo lugar, García (citado por Rodríguez, 2007) establece que "la construcción social del riesgo de desastre se refiere también a las formas en que la sociedad, en su interacción con los ecosistemas, crea nuevas amenazas" (p.92).

\section{Percepción del riesgo}

La percepción del riesgo empieza por darle una significación social, que es construida por cada individuo a partir de sus propias particularidades socio-culturales, de esa significación resultarán también las formas en que responderán al mismo.

El riesgo constituye una construcción social, en tanto las personas elaboran su significado cuando perciben un conjunto de condiciones que interpretan como peligrosas para su integridad física y sus pertenencias. De este modo, más que sobre la base de la propia situación geológica, el riesgo se construye en base a la percepción de dicha situación y a su interpretación desde la óptica del presidente, lo cual implica controlarlo, reconstruirlo, resignificarlo y ejecutar acciones para enfrentarlo (Panza \& Wiensenfeld, 1997, p.96).

La percepción del peligro de amenazas socionaturales presenta una gran variabilidad de una comunidad a otra, dependiendo de numerosos factores entre los que se incluyen la exposición continua, experiencias pasadas, magnitud y amplitud del desastre, situación socioeconómica, creencias, 
mitos, religión, conocimiento e información sobre riesgo, factores demográficos, etc., sobre la base de esos factores se produce la percepción, interpretación, valoración de la probabilidad de desastres y las formas de respuesta a las amenazas.

\section{La vulnerabilidad en la construcción social del riesgo}

"El Paradigma de la vulnerabilidad" establece que la vulnerabilidad es el resultado de procesos sociales que se derivan de los modelos de crecimiento y cambio social asumidos en distintos países (Llavel, 2000). El mismo autor continúa señalando; "Si la vulnerabilidad constituye un componente estructural de los modelos, entonces, sin cambios fundamentales, es inevitable que los desastres sigan manifestándose, probablemente con mayores impactos en el futuro" (Llavel, 2000, p. 11). Sin embargo, no sólo los modelos de crecimiento adoptados en los diferentes países influyen sobre la vulnerabilidad también intervienen:

La experiencia de los individuos en materia de exposición a los riesgos o siniestros, su nivel de preparación para la adopción de medidas preventivas, así como el comportamiento en caso de siniestro, intervienen considerablemente sobre la vulnerabilidad de las poblaciones expuestas (Chardon, 1997, p.5).

La vulnerabilidad la definen los investigadores en dependencia del enfoque interpretativo asumido, resaltando su carácter social o bien su carácter técnico, para el propósito de esta investigación es preciso resaltar el carácter social de la vulnerabilidad al hacer énfasis en elementos culturales, ideológicos e institucionales.

\section{Estrategias de adaptación social al riesgo de desastre}

A través de la historia los pueblos como las demás especies animales y vegetales que han poblado la Tierra han debido auto-ajustarse a las condiciones cambiantes de los territorios que habitan, las sociedades humanas en particular desarrollaron procesos sociales de adaptación cultural al entorno natural conquistando aun las regiones más inhóspitas del planeta. De igual manera en todas las latitudes los pueblos y comunidades desarrollan estrategias de adaptación a las amenazas y desastres.
Los pueblos se auto-ajustan a los ritmos de la naturaleza como si fueran nómadas balseros, de inundación en inundación y de diluvio en diluvio... El territorio vegetal, acuático y pluricultural se autoajusta frente a cada cambio. Se puede especular que las dinámicas combinadas de deslizamientos, inundaciones, pueblos y animales nómadas, del sol y de la lluvia, conducen a la enorme variedad y endemismo de especies, a estrategias de cambios rápidos y adaptaciones silenciosas. (Maskrey \& Velásquez, 1994, p.3)

El dinamismo de las condiciones climáticas y geomorfológicas de la Tierra ha contribuido para que los grupos sociales formulen acciones y mecanismos de adaptación a los cambios o alteraciones recurrentes de sus entornos. "Se entiende como estrategias de adaptación social al riesgo el desarrollo de acciones colectivas planeadas, para impedir, evitar o resistir el desencadenamiento de amenazas, o reducir la vulnerabilidad social subyacente" (León \& Lavell, 1996, p.60).

\section{Materiales y métodos}

El estudio se realizó en la colonia Puerto Príncipe, en el mismo lugar de habitación de los informantes clave y donde ocurren los eventos que han merecido esta indagación. La investigación es de tipo transversal dado que se realizó en un periodo específico, en este caso, en el segundo semestre del 2013.

La población objeto de análisis estuvo conformada por líderes de diferentes organizaciones, hombres y mujeres de colonia Puerto Príncipe. La muestra la integraron 19 personas, 5 mujeres y 14 hombres, fue de tipo no probabilística, y seleccionados con criterios a conveniencia del investigador. En este estudio se utilizaron la entrevista, los grupos focales y la observación como instrumentos y técnicas de recolección de información.

\section{Resultados y discusión}

\section{Construcción social del riesgo de desastre}


Numerosos factores socioculturales intervienen en la construcción del riesgo de desastre, de las experiencias expuestas durante los grupos focales y las entrevistas se recolectó abundante información no sólo acerca de la dinámica de las inundaciones, la variabilidad en la duración de los eventos de inundación, sino también sobre el fatalismo y la resignación de los pobladores frente al riesgo, la falta de respuesta efectiva a las advertencias y alertas por amenazas socionaturales, la realización de acciones modestas y poco eficaces de respuesta a las amenazas, los daños provocados en la propiedad, los efectos colaterales y la complejidad de la dinámica de las inundaciones.

\section{Creencias sobre el origen de las inundaciones}

En la comunidad se han tejido y transmitido oralmente algunas creencias que tratan de explicar el origen de las inundaciones, estas creencias están fundadas en la observación periódica de fenómenos naturales que han ocurrido a lo largo de un extenso período de tiempo, por tal razón no deben ser tomadas a la ligera sino como insumos y aportes valiosos para esta investigación valoradas como conocimiento ecológico o conocimiento ancestral.

Los boteros (gobernadores de botes) cuentan que al juntarse las aguas del río Chiquito y el río Serrano se forma una espuma pequeña, si el espumaraje del agua es poco, la llena será mediana, pero si la espuma es bastante en el encuentro de las aguas de ambos ríos, se va a producir una llena grande... Cuando se encuentran los vientos del Este y del Oeste es un encuentro terrible para Puerto Príncipe porque se va a desatar un peligroso temporal. (Líder político y comunitario, hombre, 43 años, habita en la periferia de la colonia).

\section{Percepción y conocimiento del riesgo de desastre}

En la consciencia colectiva las inundaciones representan una mayor amenaza para la propiedad y en menor medida para la seguridad de las personas. Estos fueron dos de los comentarios expuestos por dos pobladores durante uno de los grupos focales.

Las propiedades son afectadas por las inundaciones porque mata el pasto, se baja alambrados, se pierde el ganado y a veces las personas también porque somos los que perecemos cualquier daño que se dé con la llena. (Dirigente liberal, hombre, 48 años, habita en la ribera sur de Caño Chiquito). No hay estabilidad, pues amenaza de peligro de muerte, pues no, pero si porque no desarrolla sus actividades normales... (Jefe sector Policía Nacional, hombre, 28 años, habita en la parte céntrica de la colonia).

Un aspecto relevante en relación a la percepción del riesgo se ciñe al hecho que tanto los comerciantes como los pobladores económicamente más vulnerables muestran igual comportamiento ante las advertencias de amenaza por inundación, cada uno de ellos se rehúsa a abandonar sus casas y ser evacuados a los sitios de albergue establecidos en la comunidad, tal actitud señala que la percepción del riesgo es igual en ambos grupos sociales predominando el temor a la pérdida de bienes materiales con independencia del tipo o la cantidad de bienes que posean las personas. Un comerciante mostró la misma actitud durante la entrevista:

Hay momentos que uno se asusta, al ver que a veces tiene cosas que perder a veces su familia, sus niños, yo lo que he hecho es sacar los niños y quedarme yo, viendo a ver que puedo salvar del negocio (Comerciante, hombre 42 años, comerciante, habitante en la ribera sur de Caño Chiquito)

La falta de conocimiento sobre amenazas socionaturales se refleja en un acentuado fatalismo que trata de explicar lo desconocido o inexplicable atribuyéndolo a factores sobrenaturales que están fuera del alcance o del control humano.

Es que no hay soluciones, ahí el que manda es Dios y si él manda lluvia aquí hay inundación (Comerciante, hombre, 27 años)... esa es cosa de la naturaleza (Dirigente político y comerciante, 49 años, hombre, habita en la ribera de Caño Chiquito).

Por la frecuencia de las inundaciones en la colonia Puerto Príncipe la población ha llegado a conocer su situación de amenaza y ha aprendido a convivir precariamente con el riesgo, un poblador describió la convivencia con el riesgo en uno de los grupos focales de la siguiente forma: 
Lo que pasa es que uno como decían ellos, ya como que se acostumbra a llevar esos golpes, esas pérdidas mas bien y también se confía uno porque hay la ayuda que están los botes, también está la ayuda de la gente humana de los líderes y la policía, el ejército; entonces, también todo eso la gente viene a agarrar confianza, entonces porque para que digamos lo que es... la gente aquí los líderes se preocupan cuando hay estos casos entonces la gente está esperanzada y uno como que se confía un poco más porque sabe que va a llegar la policía, que va a llegar el ejército, van a llegar los lideres a llegar a evacuar. (Dirigente político y comerciante, hombre, 49 años, habita en la ribera del río Caño Chiquito).

La percepción del riesgo se manifiesta según la forma en que haya sido construido por cada individuo lo que determinara también su forma de respuesta frente a las amenazas socionaturales.

\section{Contextualización de la vulnerabilidad social}

En el análisis de las vulnerabilidades se constataron diferentes contextos que incrementan el riesgo de la comunidad, especialmente las vulnerabilidades ecológicas y económicas.

Es evidente que la complejidad y particularidad de la ecología en Puerto Príncipe constituyen un factor crítico para la vulnerabilidad de la comunidad: un sistema de lagunas, pantanos y cauces naturales se encuentra diseminado por todo el interior del poblado, Caño Chiquito y el río Serrano circundan la colonia, la topografía del terreno presenta numerosas depresiones o zonas muy bajas, la alta pluviosidad del territorio y la formación de un embalse natural de las aguas, una vez que el río Serrano confluye en río Caño Chiquito, son algunos de los elementos ambientales que al aglutinarse incrementan el riesgo por inundaciones en la comunidad, durante la entrevista grupal uno de los pobladores señaló al respecto:

Puerto Príncipe se encuentra entre medio de dos ríos, el río Serrano y el río Caño Chiquito, los dos cuando empiezan a inundarse: el río Serrano hace que las aguas del río Chiquito se regresen hacia atrás... dentro del poblado existen lagunas y cauces los cuales posteriormente son llenados con las mismas aguas del río Chiquito y del río Serrano (líder político y comunitario, hombre, 43 años, habita en la periferia de la colonia).

Los pobladores expuestos a la exclusión económica son quienes se ven mayormente afectados por las inundaciones, un habitante de la colonia menciona:

por lo menos pasamos los cuatro, seis días arriba de la casa comiendo y bebiendo arriba, los que tienen cocina de gas pues medio se las juegan, pero las otras demás personas lo que hacen es irse. (Panadero, hombre, 25 años, habita en las cercanías del puente de la 19).

La vulnerabilidad de las familias para responder de forma efectiva al riesgo de desastre se explica en términos del grado de acceso a recursos económicos, materiales y técnicos necesarios para su desarrollo y el mejoramiento de la calidad de vida. La falta de acceso a esos recursos se explica por la posición que las familias pobres ocupan en la estructura social, este es el factor más importante en la construcción de la vulnerabilidad.

\section{Estrategias de adaptación social al riesgo de desastre}

Las iniciativas identificadas en la colonia Puerto Príncipe para prevenir, mitigar o resistir las amenazas de inundación son escasas, se circunscriben a unas cuantas acciones de carácter individual las cuales no se perfilan como estrategias planificadas, participativas y de carácter comunitaria.

\section{La personalidad comunitaria: solidaridad para la auto-evacuación}

Las tareas comunitarias de evacuación en caso de desastre, por lo general no son acciones estructuradas en un plan local de gestión de riesgo, si no que corresponden a respuestas espontáneas y solidarias al momento de la emergencia. Un mecanismo de adaptación social desarrollado en la comunidad es la auto-evacuación en la que se manifiesta la personalidad comunitaria, así lo afirma uno de los líderes locales durante la aplicación de la entrevista: 
[...] le sale más fácil o mejor irse donde el vecino que venirse a la escuela o al lugar de evacuación, entonces por eso se evacuan poquitos, es una forma de solidaridad entre vecinos (Hombre, 59 años, fundador de la comunidad, habita en la ribera del río).

\section{Modificación de la forma de la vivienda}

La construcción de viviendas de dos plantas es considerada por los habitantes de Puerto Príncipe como un mecanismo de respuesta a las inundaciones.

Cada quien conoce el sistema de este lugar y..., hacen sus casas de dos plantas previendo cualquier inundación, desde hace 18 años se empezó a construir así. (Hombre, 59 años, fundador de la comunidad, habita en la ribera del río).

Las sucesivas inundaciones han provocado cambios estructurales y funcionales en la comunidad, cambios que se reflejan tanto en el comportamiento y organización social como en las respuestas comunitarias a las amenazas socionaturales.

\section{Conclusiones}

La colonia Puerto Príncipe ha desarrollado desde el asentamiento de sus primeros habitantes en la zona un proceso continuo de construcción social del riesgo de desastres. La frecuencia de las inundaciones y la multiplicidad de experiencias en estos eventos han incidido en una alta prevalencia de la consciencia de riesgo con el que se convive, en el sistema de representaciones sociales se reconoce que se vive en una situación de peligro, pero se racionaliza para disminuirlo u ocultarlo.

La degradación del medio ambiente y el factor económico influyen decisivamente en la construcción de la vulnerabilidad global en la colonia Puerto Príncipe. Las actividades realizadas en la comunidad para enfrentar las amenazas socionaturales son organizativamente frágiles, espontáneas, coyunturales y de carácter individual. La modificación de la forma de las viviendas puede considerarse como una incipiente estrategia de adaptación social al riesgo.
El riesgo de desastre por inundaciones a las que se ve expuesta constantemente la población no deben ser percibidas prematuramente como un problema originado por la ubicación inadecuada de la colonia Puerto Príncipe y las condiciones climáticas de la zona, sino que deben abordarse desde una perspectiva integral que incluya también las formas de percepción del riesgo de desastre y los contextos de vulnerabilidad de sus habitantes.

\section{Lista de referencias}

Chardon, A. (1997). La percepción del riesgo y los factores socioculturales de la vulnerabilidad. Caso de la ciudad de Manizales, Colombia. Desastres y Sociedad, (8), 4-35. Recuperado de http:// www.desenredando.org/public/revistas/dys/ rdys-8-1.0-may-2-2002-TODO.pdf

Fernández, M. A. (1996). Ciudades en riesgo. Degradación ambiental, Riesgos urbanos y Desastres. Recuperado de http://www desenredando.org/public/libros/1996/cer/CERCapo2-DARDU-ene-7-2003.pdf

Llavel, A. (200o). Desastres y Desarrollo: Hacia un Entendimiento de las Formas de Construcción Social de un Desastre: El Caso del Huracán Mitch en Centroamérica. Recuperado de http://www. desenredando.org/public/articulos/200o/ dyd/DyD200o_mar-1-2002.pdf

León, P. \& Lavell, A. (1996). Comunidades urbanas en Centro América: Vulnerabilidad a Desastres. Revista Desastres y Sociedad. (7). 57-78. Recuperado de http://www.desenredando. org/public/revistas/dys/rdyso7/dys-7-1.0may-2-2002-TODO.pdf

Maskrey, A. \& Velásquez, A. (1994). Los paradigmas de la lluvia. Desastres y Sociedad. (3), 103-108. Recuperado de http://www.desenredando. org/public/revistas/dys/rdyso3/dys3-1.o-oct24-2001-TODO.pdf

Panza, R. \& Wiensenfeld, E, (1997). Especial: Psicología social y Desastres. Desastres y Sociedad. (8), 86-102. Recuperado de http:// www.desenredando.org/public/revistas/dys/ rdyso8/dys-8-1.o-may-2-2002-TODO.pdf

Rodríguez, M.J. (2007). La conformación de los "desastres naturales". Construcción social del riesgo y variabilidad climática en Tijuana, B. C. Ra Ximhai, 19(37), 83-112. Recuperado de http:// www.redalic.org/pdf/111/11112509011.pdf 